\title{
Pay-per-view Television: Consequences of a Ban*
}

\author{
Claus Thustrup Hansen \\ University of Copenhagen and EPRU ${ }^{\dagger}$ \\ Søren Kyhl \\ University of Copenhagen
}

July 1997

\begin{abstract}
In the future, a ban of pay-per-view broadcasting will arise on certain events included on national lists in each EU country as put forward in the new "Television Without Frontiers" Directive from the 16th of April 1997. This paper analyses theoretically the consequences of such ban when the alternative is financing the events purely by TV-commercials. Results on economic efficiency does not provide much reason for a ban. However, a ban increases consumer surplus which may support a ban for distributional reasons.
\end{abstract}

JEL code: L50 \& L82.

Keywords: Pay-per-view Television, Commercials, Regulation.

\footnotetext{
* We wish to thank Birgitte Sloth and Torben Tranæs for preceding discussions on the issue. Comments by Joao Ejarque, Birgit Grodal, and Stephen Martin at a seminar in Copenhagen are also gratefully acknowledged. Correspondence: E-mail: claus.thustrup.hansen@econ.ku.dk or kyhl@econ.ku.dk.

$\dagger$ The activities of EPRU (Economic Policy Research Unit) are financed through a grant from The Danish National Research Foundation.
} 


\section{Introduction}

In the last decade Europe has witnessed a huge expansion of the Television Industry and it is expected to continue into the next Century; e.g. 'Broadcasting hours in the EU are expected to rise from present levels of 1 million hours to over 3.5 million by the end of the Century.' [EU report 1997 p. 1]. This development is largely due to technological improvements (see e.g. Oreja 1997) that have increased not only the supply of broadcasting hours but also the opportunities to sell the product.

A TV broadcast is no longer a (pure) public good, i.e. non-rival and nonexcludable, because of the emergence of cable TV, scramblers, etc. that have made it possible to exclude some households from watching a broadcast. Thus, for private TV stations pay television has become an attractive alternative to pure advertising financed television and in general it is becoming increasingly common to charge households directly for watching a specific channel or a certain event. This paper concentrates only on the second type of pay television, i.e. what is called 'pay-per-view' broadcasting, for the following reasons: First, the consequences on number of channels, number of viewers, program quality, welfare, etc. of having whole channels financed by pay subscription instead of pure advertising are relative well known (see e.g. Spence \& Owen 1977 and Owen \& Wildman 1992) and a ban of pay television in general is not on the practical policy agenda. Second, although pay-per-view broadcasting is rather new in Europe, ${ }^{1}$ it will be more and more common in the future especially because of the emergence of digital broadcasting which can provide pay-per-view programs 'much more easily' (The Economist 20/4/96 p. 53). Finally, the new EU "Television without frontiers" Directive agreed upon by the Conciliation Committee of the European Parliament and the Council on April 16, $1997^{2}$ will in the future prevent pay-per-view broadcasting of certain major (mainly sporting) events occurring on national lists made by each member state. ${ }^{3}$

This paper analyses the welfare and distributional consequences of a ban of pay-per-view broadcasting. More specifically, we consider a television station that can acquire the sole broadcasting rights to an (outstanding) event and compares the outcome of selling the event on a pay-per-view basis (including commercials)

\footnotetext{
${ }^{1}$ E.g. the first pay-per-view broadcast in Denmark was a boxing fight between Larry Holmes and Brian Nielsen the 24th of January 1997.

${ }^{2}$ See e.g. internet address: http://europa.eu.int/en/comm/dg10/avpolicy/twf/160497en.html.

${ }^{3}$ Such list exists already in the UK where the 1996 Broadcasting Act prohibit pay-per-view broadcasting of 8 events, e.g. the Wimbledon Tennis Championships.
} 
with selling the event on a pure commercial basis. This seems to be the two alternatives in many cases. Matters are more complicated if public television is included as an alternative; it is hard to determine the 'amount of inefficiency' created by having a broadcast financed through the overall tax system.

A natural analytical starting point for an economic perspective must be to look at total surplus (welfare). Intuitively, it is tempting to conclude from the above discussion that a ban of pay-per-view will improve welfare simply because a TV broadcast is a non-rival good (i.e. marginal costs are zero). Not surprisingly in the light of the Theory of The Second Best this conclusion is wrong: Although pay-per-view may reduce the number of viewers watching the event it may also reduce the number of commercials which may improve economic efficiency. Our results are not much in favour of a ban on grounds of economic efficiency: (i) Two cases exist where pay-per-view yields highest welfare, namely when a broadcast cannot be financed purely by commercials and when all potential viewers watch the event. (ii) The welfare comparison is ambiguous in the general model when these constraints are non-binding but looking at specific functional forms indicates that the potential loss of a ban is much larger than the potential gain. The distributional implications of a ban are unambiguous: consumer surplus increases and the proceeds from the property right of the event decline. Thus, distributional reasons may justify a ban if it is impossible to achieve a desired division of total surplus obtained under pay-per-view.

Surprisingly, the economic consequences of pay-per-view broadcasting is relative unexplored. To our knowledge, only one paper exists on the issue, namely Holden (1993). Our analysis differ in two respects. First, Holden (1993) considers only the effects on consumer surplus whereas we also analyse effects on total surplus. Second, Holden (1993) considers the possibility of second degree price discrimination caused by allowing pay-per-view, i.e. the station obtains revenue first by selling the event to some viewers on a pay-per-view basis and later by making a pure advertising broadcast of the event to other viewers. ${ }^{4}$ Our main analysis considers instead the case where a later broadcast of the event has zero value to the consumers (e.g. what is the value of watching a football match the day after you know the result?!). We believe this to be a more natural starting point for a basic analysis of the issue. We do, however, briefly consider the ef-

\footnotetext{
${ }^{4}$ Actually, Holden (1993) calls it third degree price discrimination. However, in the analysis the station cannot separate directly the two groups. It has to maximize profits subject to an IC constraint specifying when the first group will chose the package intended for the second group. This is in fact second degree price discrimination according to the definition in Tirole (1989).
} 
fect on total surplus of including second degree price discrimination. Simulations suggest that our welfare conclusions are unchanged when including this element.

The paper is organized as follows. The next section presents the basic model and characterises the interior solutions of letting the broadcast of an event be controlled by a pure advertising station, a pay-per-view station, and a social planner. The third section compares these outcomes. The fourth section uses a specific example to indicate the relative size of total surplus, consumer surplus, and the value of the broadcasting rights under pay-per-view and pure advertising. The specific example is also used to elaborate on the consequences of second degree price discrimination. The last section discusses some policy implications of the results.

\section{A Basic Television Model}

An agent possesses the "legal" (monopoly) rights to broadcast an outstanding event. ${ }^{5}$ The agent is willing to sell the rights if the price $\Omega$ is at least $\tilde{\Omega} \geq 0 .{ }^{6} \mathrm{We}$ assume that there is infinite many television stations each biding for the rights to broadcast the event as long as they expect non-negative profits. The price of the contract will equal the highest bid. The potential viewers of the event are indexed by $x \in[0, \bar{x}]$ such that the willingness to pay of the viewers are declining in the index. The willingness to pay of the marginal viewer with no commercials is denoted by $\zeta(x)$. It is assumed that this function is not 'too convex', i.e. $\zeta^{\prime \prime}(x) x / \zeta^{\prime}(x)>-1$, which is sufficient (but not necessary) to ensure that any point satisfying the first order conditions is indeed a solution (if the solution is interior). ${ }^{7}$ All viewers have the same disutility of commercials measured in money terms,${ }^{8} \eta(a)$, which is strictly increasing and convex in the level of advertising, $a$, i.e. $\eta^{\prime}(\cdot)>0, \eta^{\prime \prime}(\cdot) \geq 0$. This implies that the inverse demand curve facing the television station for a given level of advertising takes the following separable form, $p(x, a) \equiv \zeta(x)-\eta(a)$. We assume that any solution $(x, a)$ fulfills $p(x, a) \geq 0$; this states that it is impossible to pay/subsidize a viewer to watch the event. The

\footnotetext{
${ }^{5}$ The agent could be UEFA possessing the rights to the Euro Cup Final in football or promoter Don King owning the rights to boxing matches involving Mike Tyson.

${ }^{6}$ In the case where promoter Don King owns the rights to a boxing match involving Mike Tyson this could reflect the reservation price of Mike Tyson.

${ }^{7}$ In the Appendix, it is shown that the second order conditions are satisfied for all problems under consideration.

${ }^{8}$ This represents the direct displeasure of being interrupted and 'enforced' to look at commercials net of any eventual gain (e.g. because of the informational content).
} 
station sells commercials giving a net revenue of $\psi(a)$ per viewer for a given level of advertisement; $\psi(a)$ is assumed increasing and concave, i.e. $\psi^{\prime}(\cdot)>0, \psi^{\prime \prime}(\cdot) \leq 0$. We consider two possible ways of selling the event; through pure advertising or pay-per-view. In the pure advertising case all revenues are obtained from selling commercials whereas in the pay-per-view case revenues stem from commercials as well as direct payment from the viewers. For comparison, we also consider the case of a social planner optimizing total surplus under the same constraints. In the following three subsections, we characterize the interior solutions of each case, and in the next section we will compare the three cases also taking into consideration two possible constraints that may give rise to boundary solutions; (i) the equilibrium contract price must be at least equal to the reservation price of the supplier $\tilde{\Omega}$, (ii) the number of viewers cannot exceed the potential number of viewers $\bar{x}$.

\subsection{Advertising}

The advertising monopolist is prohibited from charging the viewers and thus chooses only advertising level $a$ in order to maximize profits,

$$
\Pi(\Omega)=\psi(a) \cdot x-\Omega,
$$

subject to the following inverse relationship between the number of viewers and the amount of advertising,

$$
\zeta(x)-\eta(a)=0 .
$$

Maximization yields the following first order condition,

$$
\psi^{\prime}(a)=-\frac{\eta^{\prime}(a)}{\zeta^{\prime}(x) x} \psi(a) .
$$

Equation (2.3) states that the advertising monopolist chooses the advertising level such that marginal revenue per viewer of increased advertising (the LHS) equals marginal costs (the RHS ) consisting of reduced number of viewers from increased advertising times total advertising revenue per viewer. An equilibrium $\left(x_{A}, a_{A}, \Omega_{A}\right)$ that is interior is characterized by $(2.2),(2.3)$, and $\Pi(\Omega)=0$. 


\subsection{Pay-per-view}

The pay-per-view monopolist chooses price and advertising level in order to maximize, ${ }^{9}$

$$
\Pi(\Omega)=(\zeta(x)-\eta(a)) x+\psi(a) x-\Omega,
$$

where the first two terms represent revenues from 'pay-per-view' and commercials, respectively. Note, that a solution to this problem does not yield less profits than the advertising solution. To be an interior solution, we require a strictly positive pay-per-view price, $\zeta\left(x_{P}\right)-\eta\left(a_{P}\right)>0$, which we assume to be fulfilled in the following. The unique maximum $\left(x_{P}, a_{P}\right)$ is then characterized by the first order conditions,

$$
\begin{gathered}
\zeta(x)-\eta(a)+\zeta^{\prime}(x) x=-\psi(a), \\
\psi^{\prime}(a)=\eta^{\prime}(a) .
\end{gathered}
$$

The second condition states that the optimal level of advertising is chosen where marginal revenue per viewer equals marginal disutility per viewer. The marginal disutility per viewer is also the marginal costs of the television station as it measures how much the station has to reduce the price per viewer for a given number of viewers. The first condition is the standard marginal revenue equals marginal costs condition of a monopolist; the LHS represents the marginal revenue from pay-per-view payments of getting one more viewer equal to the price per viewer (the first two terms) and the loss in revenue from reducing the price in order to get one more viewer; the RHS represents marginal revenue from advertising of getting one more viewer, i.e. negative marginal costs. ${ }^{10}$ An equilibrium $\left(x_{P}, a_{P}, \Omega_{P}\right)$ that is interior is characterized by $(2.5),(2.6)$, and $\Pi(\Omega)=0$.

\footnotetext{
${ }^{9}$ We disregard any direct costs of charging the viewers. Such costs are relative low and will probably be infinitesimal in a future with Digital Broadcasting.

${ }^{10}$ Thus, an interior solution lies always on the inelastic part of the demand curve provided that such area exists. If not the number of viewers equals the upper bound $\bar{x}$ which is analysed in the next section.
} 


\subsection{Social Planner}

For comparison, we derive the outcome of a social planner that chooses $(x, a)$ to maximize total surplus defined as ${ }^{11}$

$$
W \equiv \int_{0}^{x} \zeta(s) d s-\eta(a) x+\psi(a) x-\tilde{\Omega},
$$

Maximizing (2.7) taking into consideration the constraint $\zeta(x)-\eta(a) \geq 0$ yields the unique solution $\left(x_{S}, a_{S}\right)$ satisfying the following conditions,

$$
\begin{gathered}
\zeta(x)-\eta(a)=0, \\
\psi^{\prime}(a)=-\frac{\eta^{\prime}(a)}{\zeta^{\prime}(x) x} \psi(a)+\eta^{\prime}(a) .
\end{gathered}
$$

The first condition states that the socially optimal pay-per-view price is zero, i.e. the marginal costs of one more viewer is zero. The second condition is identical to the advertising solution (2.3) except from the last term which represents the increased disutility to the viewers of a higher advertising level. This effect was not taking into account by the advertising monopolist thus creating a negative externality on the viewers. A solution $\left(x_{S}, a_{S}\right)$ that is interior is characterized by (2.8) and (2.9).

\section{Pay-per-view vs. Pure Advertising}

In this section, we compare the outcome of the two institutions. In doing so, we want to take into consideration the possibility of boundary solutions. Two natural constraints may give rise to boundary solutions; $(i)$ the equilibrium contract price must be at least equal to the reservation price of the supplier, i.e. $\Omega \geq \tilde{\Omega}$; (ii) the solution must imply that the number of viewers is less than the potential number of viewers, i.e. $x \leq \bar{x}$. We start by focusing on interior solutions where these constraints are not binding. It is probably quite intuitive that the advertising

\footnotetext{
${ }^{11}$ In this definition, it is assumed that there exists a direct welfare gain of having commercials equal to the difference between revenue from commercials and viewer disutility of commercials. One might argue that the revenue does not contribute with any surplus or even that we should add a negative term representing further negative effects; e.g. because seemingly identical products are 'diversified' by commercials thereby reducing the competitiveness among firms buying advertising time. Including such effects would strengthen our overall conclusion concerning welfare.
} 
level will be highest in the case of pure advertising when comparing the pay-perview, advertising, and socially optimal solution. It is, however, less clear both intuitively and analytically that the number of viewers will be lowest with payper-view as the advertising level may be so large in the pure advertising case that marginal consumers prefer the pay-per-view price in combination with a lower advertising level. To deal with this ambiguity, it is useful to rewrite and combine the first order conditions of the previous section to get the following relationships

$$
\begin{gathered}
\zeta\left(x_{P}\right)+\zeta^{\prime}\left(x_{P}\right) x_{P}=-\left[\psi\left(a_{P}\right)-\eta\left(a_{P}\right)\right], \\
\zeta\left(x_{A}\right)+\zeta^{\prime}\left(x_{A}\right) x_{A}=-\left[\frac{\eta^{\prime}\left(a_{A}\right)}{\psi^{\prime}\left(a_{A}\right)} \psi\left(a_{A}\right)-\eta\left(a_{A}\right)\right], \\
\zeta\left(x_{S}\right)+\zeta^{\prime}\left(x_{S}\right) x_{S}=-\left[\frac{\eta^{\prime}\left(a_{S}\right)}{\psi^{\prime}\left(a_{S}\right)-\eta^{\prime}\left(a_{S}\right)} \psi\left(a_{S}\right)-\eta\left(a_{S}\right)\right],
\end{gathered}
$$

where (3.1) follows directly by isolating the $x$ terms in the pay-per-view equation (2.5); (3.2) are derived by rewriting and adding the advertising equations (2.2) and (2.3); (3.3) are derived by rewriting and adding the equations (2.8) and (2.9) which characterize the socially optimal solution. Using these equations and the equations determining the advertising level (i.e. (2.3), (2.6), and (2.9), respectively), makes it possible to illustrate the comparison graphically. This is done in Figure 1 where panel a illustrates the result of comparing number of viewers $x$ and panel $\mathrm{b}$ the result of comparing advertising levels $a$.

\section{(Figure 1 here)}

The pay-per-view solution is intuitively straightforward in this diagram; $a_{P}$ maximize the difference between $\psi(a)$ and $\eta(a)$ and $x_{P}$ is set where marginal revenue (before advertising, i.e. $\eta(a)$ is moved to the RHS) equals marginal costs. The two other solutions are more complicated; the LHS of (3.2) and (3.3) does not represent marginal revenue but instead the marginal aggregate pay willingness of the viewers (before disutility of advertising). The shadow value of a change in the aggregate willingness to pay, $[\zeta(x)-\eta(a)] x$, is one in the case of pay-perview (as it equals the revenue from pay-per-view payments, $p_{P} x_{P}$ ) but $\frac{\psi^{\prime}\left(a_{A}\right)}{\eta^{\prime}\left(a_{A}\right)}$ and $\frac{\psi^{\prime}\left(a_{S}\right)-\eta^{\prime}\left(a_{S}\right)}{\eta^{\prime}\left(a_{S}\right)}$ in the two other cases, respectively. In the advertising case it transforms a loss in aggregate pay willingness into a loss in advertising revenue necessary to obtain an increase in the number of viewers. In the case of the social planner it transforms a loss in aggregate pay willingness into a loss/gain in 
aggregate welfare from the reduction in advertising level necessary to obtain an increase in the number of viewers. The inverse of these shadow values occur at the RHS of (3.1) - (3.3) and are the only thing separating the equations from each other. This feature is used below when proving the outcome illustrated in Figure 1 and stated in following theorem,

Theorem 3.1. Assume that the equilibrium contract price with pure advertising is larger than or equal to the reservation price and that number of viewers under pay-per-view is less than the potential number of viewers. Then pay-per-view implies less viewers than pure advertising which implies less viewers than the socially optimal solution. Pay-per-view involves an intermediate level of commercials; less than pure advertising but more than the socially optimal solution. The socially optimal solution has highest welfare level; but the outcome of a welfare comparison of pay-per-view versus pure advertising is indeterminable. Formally: If $\Omega_{A} \geq \tilde{\Omega}$ and $x_{P}<\bar{x}$ then $x_{P}<x_{A}<x_{S}, a_{S}<a_{P}<a_{A}$, and $\max \left(W_{A}, W_{P}\right)<W_{S}$.

Proof. Note that $\Omega_{A} \geq \tilde{\Omega}$ implies $\Omega_{P} \geq \tilde{\Omega}$. We start by deriving the result when the constraint $x_{A} \leq \bar{x}$ is not binding. First, we compare the advertising solution $\left(x_{A}, a_{A}\right)$ and the pay-per-view solution $\left(x_{P}, a_{P}\right)$. If $H(a) \equiv \psi(a) \frac{\eta^{\prime}(a)}{\psi^{\prime}(a)}-\eta(a)$ then it follows from the concavity of $\psi(\cdot)$, the convexity of $\eta(\cdot)$, and the equation (2.6) that $H\left(a_{P}\right)=\psi\left(a_{P}\right)-\eta\left(a_{P}\right)$ and $H(a)>\psi\left(a_{P}\right)-\eta\left(a_{P}\right) \Leftrightarrow a>a_{P}$. The LHS of (3.1) and (3.2) are declining in the argument yielding the implication,

$$
x_{P}<x_{A} \Leftrightarrow a_{P}<a_{A} .
$$

We will now establish that $x_{P}<x_{A}$. Assume the opposite is true, $x_{P} \geq x_{A}$ and therefore also that $a_{P} \geq a_{A}$. The reservation price of the marginal viewer, $p(x, a) \equiv \zeta(x)-\eta(a)$, will then be characterized by $p\left(x_{P}, a_{P}\right) \leq p\left(x_{A}, a_{A}\right)=0$ which contradicts the fact that there exists an interior solution to the pay-perview problem, i.e. $p\left(x_{P}, a_{P}\right)>0$. Thus, $x_{P}<x_{A}$ and $a_{P}<a_{A}$. The inequality, $a_{S}<a_{P}$, follows directly by comparing (2.6) with (2.9). That $x_{A}<x_{S}$ follows from $a_{S}<a_{P}$ and the equations (2.2) and (2.8). The welfare result follows from the fact that both monopolists choose higher advertising level than the social planner and that the social planner maximizes under the same constraints as the pay-per-view monopolist. Until now we have skipped the possibility that the constraint $x_{A} \leq \bar{x}$ is binding. However, it is clear from Figure 1 that the results are still valid for this case as $x_{P}<\bar{x}$. 
Thus, if an event can be financed by pure advertising and the constraint on the number of viewers is non-binding with pay-pay-view then a ban of pay-per-view broadcasting will increase the number of viewers towards the socially optimal solution but also increase the advertising level further away from the socially optimal solution. Therefore, the effect on total surplus is indeterminable. We will further elaborate on this welfare comparison in the next section.

Readers familiar with the Swan invariance theorem (see e.g. Tirole 1989 p. 102) may be puzzled by the conclusion that the pay-per-view monopolist advertise more than socially optimal; the aggregate disutility of commercials, $\eta(a) x$, act as linear 'production' costs in the profit expression which normally result in an efficient choice of $a$. This does not occur in our case because of the assumption that it is impossible to subsidize viewers to watch an event, $p(x, a) \geq 0$. The pay-per-view advertising level would coincide with the social planners if the social planner could spend some of the proceeds from advertising to subsidize people normally unwilling to watch the event. ${ }^{12}$

The above theorem included one boundary solution, namely when the advertising solution was constrained by the number of potential viewers. The following theorem covers the remaining possibilities,

Theorem 3.2. Assume either that it is impossible to finance an event through pure advertising or that the constraint on the number of viewers is binding with per-per-view. Then pay-per-view will yield larger welfare than pure advertising. Formally: If $\Omega_{A}=0$ or $x_{P}=\bar{x}$ then $W_{P} \geq W_{A}$.

Proof. Clearly, if $\Omega_{A}=0$ and $x_{P} \leq \bar{x}$ then one of two things may happen; a) pay-per-view is profitable and yields positive total surplus; $b$ ) pay-perview is not profitable and thus the event will not be broadcasted at all. If $x_{P}=\bar{x}$ and $\Omega_{A}>\tilde{\Omega}$ then $x_{A}=x_{P}=\bar{x}$. Now, it follows from (2.7), that $W_{A} \geq W_{P} \Leftrightarrow \eta\left(a_{A}\right)-\eta\left(a_{P}\right) \geq \psi\left(a_{A}\right)-\psi\left(a_{P}\right)$. One of two things may happen: Let $\tilde{a}$ be defined by $\eta^{\prime}(\tilde{a})=\psi^{\prime}(\tilde{a})$. a) If $a_{A}>\tilde{a}$ then $a_{P}=\tilde{a}<a_{A}$ and thus $W_{P}>W_{A}$. b) If $a_{A}=\tilde{a}$ then $a_{P}=a_{A}=\tilde{a}$ implying that $W_{P}=W_{A}$.

\footnotetext{
${ }^{12}$ Graphically, the number of viewers, $x_{S}$, would be determined in Figure 1a by setting the willingness to pay of the marginal viewer, $\zeta(x)$, equal to net marginal costs $\eta\left(a_{P}\right)-\psi\left(a_{P}\right)$. Obviously, such solution is characterized by a negative willingness to pay of the marginal viewer, $\zeta\left(x_{S}\right)$, implying a contract specifying that the viewer will watch the event in exhange of money. We assume that such contracts are impossible to enforce.
} 
The intuition of these results are straightforward: Pay-per-view can finance events that are not profitable by pure advertising thus giving some surplus instead of none. When the pay-per-view solution is constrained by the number of potential viewers so is the advertising solution. Thus, the number of viewers is identical in which case pay-per-view yields the largest welfare because of a lower advertising level (cf. Figure 1b). So far we have only considered effects on total surplus. Turning to distributional implications, it is already clear that pay-per-view yields a larger contract price (i.e. transfer from the TV station to the owner of the event) than pure advertising, $\Omega_{P} \geq \Omega_{A}$. Furthermore, we have,

Corollary 3.3. Assume it is possible to finance an event through pure advertising. Then pure advertising yields larger consumer surplus than pay-per-view.

Proof. The consumer surplus equals

$$
\begin{gathered}
C S_{A} \equiv \int_{0}^{x_{A}}\left[\zeta(s)-\eta\left(a_{A}\right)\right] d s=\int_{0}^{x_{A}}\left[\zeta(s)-\zeta\left(x_{A}\right)\right] d s, \\
C S_{P} \equiv \int_{0}^{x_{P}}\left[\zeta(s)-\eta\left(a_{P}\right)-p\right] d s=\int_{0}^{x_{P}}\left[\zeta(s)-\zeta\left(x_{P}\right)\right] d s,
\end{gathered}
$$

with pure advertising and pay-per-view, respectively. From Theorem 1 and Theorem 2 , we have $x_{A} \geq x_{P} \Rightarrow C S_{A} \geq C S_{P}$.

This result is also clear from Figure 1. The consumer surplus under pay-perview equals the triangle bounded by the $\zeta(x)$ - curve and the horizontal line going through point $\mathbf{P}$. Similarly, the consumer surplus under advertising equals the triangle bounded by the $\zeta(x)$ - curve and the horizontal line going through point A. This result strengthens the overall conclusion in Holden (1993) which showed (using numerical examples) that pay-per-view may involve a large loss of consumer surplus.

\section{A Useful Example}

Only one comparison in the preceding analysis yields an ambiguous result, namely the welfare comparison of pay-per-view versus advertising in Theorem 1 . In this 
section, we use a simple example with specific functional forms to elaborate further on this part of Theorem 1 and furthermore to give an impression of the possible magnitudes of total surplus, consumer surplus, and contract price. This section also addresses the possible welfare consequences of second degree price discrimination which may arise when pay-per-view is allowed. So far, the analysis has not dealt with this issue as it turns out to be difficult to derive any clear results using the level of generality of the preceding analysis. In this section, we do some numerical simulations based on the specific example in order to indicate how this element would alter the previous conclusions concerning welfare.

Our specific example is based on the following functional forms: The willingness to pay without commercials is described by $\zeta(x)=\alpha-\beta x$, disutility of commercials by $\eta(a)=\gamma a$, and revenue from commercials by $\psi(a)=\sqrt{a}$ where $\alpha, \beta$, and $\gamma$ are strictly positive parameters. Calculating the optimal solution $\left(x_{A}, a_{A}\right)$ and inserting this into (2.7), (3.4), and (2.1) yields welfare level, consumer surplus, and equilibrium contract price under advertising:

$$
W_{A}=\frac{2 \alpha}{3 \beta}\left(\frac{\alpha}{3}+\sqrt{\frac{\alpha}{3 \gamma}}\right)-\tilde{\Omega}, C S_{A}=\frac{x_{A}^{2}}{2}, \Omega_{A}=\frac{2 \alpha}{3 \beta} \sqrt{\frac{\alpha}{3 \gamma}} \quad \text { for all } \alpha, \beta, \gamma
$$

The pay-per-view case has to obey the condition $p(x, a)>0$ yielding the following parameter restriction $\gamma>3 /(4 \alpha)$. When this condition is violated the pay-perview monopolist chooses price equal to zero and same advertising level as the advertising monopolist, i.e. the above solution. When the condition is fulfilled, welfare level, consumer surplus, and equilibrium contract price under pay-per-view are calculated using $(2.7),(3.5)$, and (2.4):

$$
W_{P}=\frac{3}{8 \beta}\left(\alpha+\frac{1}{4 \gamma}\right)^{2}-\tilde{\Omega}, C S_{P}=\frac{x_{P}^{2}}{2}, \Omega_{P}=\frac{(4 \alpha \gamma+1)^{2}}{64 \beta \gamma^{2}} \quad \text { for } \gamma>3 /(4 \alpha) \text {. }
$$

To compare the two institutions, we use the following expressions

$$
\begin{gathered}
f(\alpha, \gamma) \equiv \frac{W_{P}+\tilde{\Omega}}{W_{A}+\tilde{\Omega}}=\frac{9\left(\alpha+\frac{1}{4 \gamma}\right)^{2}}{16 \alpha\left(\frac{\alpha}{3}+\sqrt{\frac{\alpha}{3 \gamma}}\right)} \quad \text { for } \gamma>3 /(4 \alpha), \\
g(\alpha, \gamma) \equiv \frac{C S_{P}}{C S_{A}}=\left(\frac{3}{4}+\frac{3}{16 \gamma \alpha}\right)^{2} \quad \text { for } \gamma>3 /(4 \alpha),
\end{gathered}
$$




$$
h(\alpha, \gamma) \equiv \frac{\Omega_{P}}{\Omega_{A}}=\frac{3(4 \alpha \gamma+1)^{2}}{128 \gamma^{2} \alpha \sqrt{\frac{\alpha}{3 \gamma}}} \quad \text { for } \gamma>3 /(4 \alpha),
$$

where $f(\alpha, \gamma)$ denotes relative welfare, $g(\alpha, \gamma)$ relative consumer surplus, and $h(\alpha, \gamma)$ relative contract price. Pay-per-view yields the largest (lowest) welfare, consumer surplus, contract price when $f(\alpha, \gamma), g(\alpha, \gamma)$, and $h(\alpha, \gamma)$ is larger (lower) than one, respectively. It is clear from these formulas that each comparison are independent of $\beta$, i.e. the slope of $\zeta(x)$. Thus, the comparisons can be described in a two dimensional parameter space as done in Figure 2.

\section{(Figure 2 here)}

In Figure 2 the parameter space is partitioned into 3 areas. In the black area, the constraint $p(x, a)>0$ is violated and the pay-per-view solution is identical to the advertising solution. In the white area the pay-per-view price is positive and advertising yields a larger welfare level than pay-per-view. In the gray area the pay-per-view price is positive and pay-per-view is welfare superior to advertising. ${ }^{13}$ In both the white and gray area pay-per-view yields larger contract price and lower consumer surplus than advertising. Turning to magnitudes, it is useful to note that $f(\alpha, \gamma), g(\alpha, \gamma)$, and $h(\alpha, \gamma)$ are constant when considering combinations of $\alpha$ and $\gamma$ fulfilling $\gamma=\rho / \alpha$ where $\rho$ is a positive constant. ${ }^{14}$ Thus, any hyperbola in the white and gray area represents an iso-relative welfare, consumer surplus, and contract price curve. The figures on the $45^{\circ}$-line in Figure 2 equal the values of $f(\alpha, \gamma)$ on the hyperbolas going trough these points and the figures in parentheses equal $g(a, \gamma)$ and $h(\alpha, \gamma)$, respectively. In the white area, we have chosen the lowest value of $f(\alpha, \gamma)$; thus allowing pay-per-view in this area will at most give a welfare loss equal to $5 \%$. In the gray area the values of $f(\alpha, \gamma)$ continue to increase as we move north-east in the diagram and the figures show that a welfare improvement of up to $43 \%$ is possible if pay-per-view is allowed in this area. ${ }^{15}$ Looking at distributional implications, Figure 2 reveals larger relative contract

\footnotetext{
${ }^{13}$ The Figure only shows a small part of the entire parameter space $(\alpha, \gamma) \in \mathbb{R}^{2}$. If we consider the parameter space $(\alpha, \gamma) \in[0, h]^{2}$ when $h$ goes to infinity, it is easy to show that the white area's share converges to 0 whereas the gray area's share converges to 1 .

${ }^{14}$ Just insert $\gamma=\rho / \alpha$ into (4.1), (4.2), and (4.3) and note that the formulas become independent of $\alpha$.

${ }^{15}$ Considering the entire parameter space, $(\alpha, \gamma) \in \mathbb{R}^{2}$, it is possible to show that the welfare improvement is bounded by the value $68 \frac{3}{4} \%$. This maximum occurs when considering $f(a, \gamma)$ at the hyperbolas $\gamma=\rho / \alpha$ for $\rho \rightarrow \infty$.
} 
price and lower relative consumer surplus when moving north-east in the diagram. The consumer surplus may decrease by $43 \%$ and the contract price may increase by $501 \%$ if pay-per-view is allowed.

We end this section by briefly considering the welfare implications of second degree price discrimination caused by allowing pay-per-view broadcasting. As mentioned in the introduction, Holden (1993) analysed this issue but looked only at distributional consequences. Now, we assume that the television station can broadcast two versions of the event. The first broadcast (usually live) is sold on a pay-per-view basis as in the previous analysis and the later broadcast (usually the day after) on a pure advertising basis. Viewers obtain lower utility from watching the second version, e.g. because the news content is gone. Following Holden (1993), we assume that viewer's willingness to pay for the second version equal $\lambda \zeta(\cdot)$ where $\lambda \in(0,1)$. The television station chooses pay-per-view price $p$, advertising level in the first version $a_{1}$, and advertising level in the second version $a_{2}$ in order to maximize the following,

$$
\Pi\left(p, a_{1}, a_{2}\right)=p x_{1}+\psi\left(a_{1}\right) x_{1}+\psi\left(a_{2}\right) x_{2}-\Omega,
$$

subject to the incentive compatibility constraint (IC),

$$
\zeta\left(x_{1}\right)-\eta\left(a_{1}\right)-p \geq \lambda \zeta\left(x_{1}\right)-\eta\left(a_{2}\right),
$$

and the participation constraint (PC),

$$
\lambda \zeta\left(x_{1}+x_{2}\right)-\eta\left(a_{2}\right) \geq 0,
$$

where $x_{2}$ denotes the number of viewers watching the second version. The IC states that the viewers watching the first version most obtain higher utility than what they can get by watching the second version. The PC states that only households obtaining positive utility view the broadcast. This maximization program is solved in Appendix using the same functional forms as above. Let $z(\alpha, \gamma, \lambda)$ denote the welfare under pay-per-view with 2 . degree price discrimination relative to welfare under pure advertising. Like $f(\alpha, \gamma)$ this function has constant value along any hyperbola $\gamma=\rho / \alpha$ for a given value of $\lambda$. Therefore, we will proceed by only comparing $z(\alpha, \gamma, \lambda)$ and $f(\alpha, \gamma)$ on the $45^{0}$-line for a given value of $\lambda$. This yields the outcome illustrated in Figure 3 where the solid curve illustrates the value of $f(\alpha, \gamma)=z(\alpha, \gamma, 0)$ whereas the dashed curves illustrate $z(\alpha, \gamma, \lambda)$ 
for specific choices of $\lambda^{16}$

\section{(Figure 3 here)}

Starting from left, the horizontal line at one corresponds to the black area in Figure 2. The part of the curves below one corresponds to the white area and the part above one corresponds to the gray area. The basic conclusion from Figure 3 is that an inclusion of second degree price discrimination does not contribute with any significant changes to the preceding results. Allowing pay-per-view enlarges the rent capacity of the event in two ways; first by allowing to charge a price and second by admitting second degree price discrimination. Our example indicates that the first effect is by far the largest.

\section{Policy Implications}

The purpose of the new "Television Without Frontiers" Directive is indicated by the following citation: 'The European Parliament has insisted recently, on several occasions, on legal intervention with a view to ensuring that the viewing public can continue to have access to such events on live, in-the-clear, TV.' [Oreja 1997 p. 2]. ${ }^{17}$ Thus, there is basically two goals: first, access for everybody to major events on live TV and second, that access shall be on non pay television. The analysis of this paper addresses only the second issue. That is we ask the question: Should pay-per-view be banned given that everybody has access? ${ }^{18}$ Looking at total welfare does not provide much reason for a ban. A ban reduces welfare if it is impossible to finance the event through pure advertising or if all potential viewers are watching the event under pay-per-view (Theorem 2). Otherwise, a ban may or may not reduce welfare (Theorem 1) and specific functional forms indicate that a ban may reduce welfare by up to $68 \%$ whereas it can only improve welfare by at most $5 \%$. In the analysis, commercials is treated very favorable by including commercial revenue in total surplus although it may be questionable

\footnotetext{
${ }^{16}$ The dashed curves are all above the solid horizontal line in a small region around $\alpha=\gamma=$ 0.85. This occurs because the constraint $p \geq 0$ is binding in the basic pay-per-view case but not in the case with price discrimination. The dashed curves all coincide with the solid curve after some threshold because it is not profitable to broadcast the second version. Thus, the solution coincides with the simple pay-per-view case.

${ }^{17}$ Note, that in-the-clear television includes pure advertising financed broadcasting.

${ }^{18}$ In a near future with digital broadcasting access will probably not be an issue anymore.
} 
whether this revenue represents a direct welfare gain (cf. footnote 12). A less favorable treatment would further strengthen the overall welfare conclusion.

There might be other reasons supporting a ban. If it is impossible to obtain the desired division of total surplus, policy makers might favour a ban for distributional reasons. Thus, a ban may arise because policy makers are most concerned with the welfare of the viewers as our results state that a ban unambiguously increases consumer surplus (Corollary 1). A ban reduces the contract price, i.e. the proceeds to the initial owner of the broadcasting rights. Figure 2 indicates increases of up to $43 \%$ in consumer surplus and reductions in contract price of up to $501 \%$.

If a ban is chosen for distributional reasons it is important to recognize that it involves a risk of a large deadweight loss unless one has precise knowledge of structural relations. Thus, it may be worthwhile to consider whether distributional goals can be obtained through a tax on the television station's proceeds from pay-per-view broadcasting of certain events instead of prohibiting pay-perview broadcasting entirely.

The large effect on the contract price may lead to another worry of the new Directive: it creates big incentives for (sporting) organizations to lobby in order not to be included on the national lists. Thus, it is not clear that the political decision process results in a desirable outcome. Furthermore, it seems paradoxically that (sporting) organizations may be punished economically after great success that suddenly makes their (sporting) events of national interest. That is, it is beneficial to be popular but not too popular.

\section{References}

[1] EU report, 1997, 'Audiovisual Policy', web-site http://europa.eu.int/pol/ av/en/av.htm.

[2] Holden, S, 1993, 'Network or pay-per-view?', Economics Letters, 43, pp. 59-64.

[3] Oreja, M., 1997, 'Communication from Mr. Oreja to the Commission. Exclusive Rights for TV Broadcasting of Major (Sports) Events', web-site http://europa.eu.int/en/record/other/tven.htm.

[4] Owen, B. M. \& Wildman, S. S., 1992, 'Video Economics', Cambridge: Harvard University Press. 
[5] Spence, M. \& Owen, B., 1977, 'Television Programming, Monopolistic Competition, and Welfare', Quarterly Journal of Economics, 91, pp. 103-126.

[6] Tirole, J., 1989, 'The Theory of Industrial Organization', Cambridge: MIT Press.

\section{A. Appendix}

\section{A.1. Second order condition}

\section{A.1.1. Advertising}

From the definition of profits, we get the following second order condition

$$
\Pi_{a a}=\psi^{\prime \prime}(a) x+\frac{\psi^{\prime}(a) \eta^{\prime}(a)+\psi(a) \eta^{\prime \prime}(a)}{\zeta^{\prime}(x)}+\frac{d x}{d a}\left(\psi^{\prime}(a)-\psi(a) \cdot \frac{\eta^{\prime}(a) \zeta^{\prime \prime}(x)}{\left(\zeta^{\prime}(x)\right)^{2}}\right)<0
$$

Inserting $\frac{d x}{d a}$ derived from (2.2) and rearranging give

$$
\psi^{\prime \prime}(a) x^{2}+\frac{\psi^{\prime}(a) \eta^{\prime}(a) x+\psi(a) \eta^{\prime \prime}(a) x}{\zeta^{\prime}(x)}+\frac{\eta^{\prime}(a)}{\zeta^{\prime}(x)}\left(\psi^{\prime}(a) x-\frac{\psi(a) \eta^{\prime}(a)}{\zeta^{\prime}(x)} \cdot \frac{\zeta^{\prime \prime}(x) x}{\zeta^{\prime}(x)}\right)<0
$$

This is fulfilled if

$$
\psi^{\prime}(a) x-\frac{\psi(a) \eta^{\prime}(a)}{\zeta^{\prime}(x)} \cdot \frac{\zeta^{\prime \prime}(x) x}{\zeta^{\prime}(x)}>0 .
$$

It is seen from (2.3) that the assumption $\frac{\zeta^{\prime \prime}(x) x}{\zeta^{\prime}(x)}>-1$ is sufficient to ensure that this is satisfied for $\left(x_{A}, a_{A}\right)$ which is then the unique solution to the maximization problem.

\section{A.1.2. Pay-per-view}

Setting up the Hessian matrix gives

$$
\left(\begin{array}{cc}
\Pi_{x x} & \Pi_{x a} \\
\Pi_{x a} & \Pi_{a a}
\end{array}\right)=\left(\begin{array}{cc}
2 \zeta^{\prime}(x)+\zeta^{\prime \prime}(x) x & -\eta^{\prime}(a)+\psi^{\prime}(a) \\
-\eta^{\prime}(a)+\psi^{\prime}(a) & -\eta^{\prime \prime}(a) x+\psi^{\prime \prime}(a) x
\end{array}\right) .
$$

Using the first order conditions, we have

$$
\left(\begin{array}{ll}
\Pi_{x x} & \Pi_{x a} \\
\Pi_{x a} & \Pi_{a a}
\end{array}\right)=\left(\begin{array}{cc}
2 \zeta^{\prime}\left(x_{P}\right)+\zeta^{\prime \prime}\left(x_{P}\right) x_{P} & 0 \\
0 & -\eta^{\prime \prime}\left(a_{P}\right) x_{P}+\psi^{\prime \prime}\left(a_{P}\right) x_{P}
\end{array}\right)
$$


yielding the conditions,

$$
\begin{gathered}
2 \zeta^{\prime}\left(x_{P}\right)+\zeta^{\prime \prime}\left(x_{P}\right) x_{P}<0 \\
-\eta^{\prime \prime}\left(a_{P}\right)+\psi^{\prime \prime}\left(a_{P}\right)<0
\end{gathered}
$$

The assumption $\frac{\zeta^{\prime \prime}(x) x}{\zeta^{\prime}(x)}>-1$ is sufficient to ensure that the first condition is fulfilled whereas the second is fulfilled by the properties of the functions $\eta(\cdot)$ and $\psi(\cdot)$. Thus, provided that there is an interior point satisfying the first order conditions, we know this is the unique solution to the maximization problem.

\section{A.1.3. Social planner}

From (2.7), we get the following second order condition

$$
\begin{aligned}
W_{a a}= & \frac{\eta^{\prime \prime}(a) \psi(a)+\eta^{\prime}(a) \psi^{\prime}(a)}{\zeta^{\prime}(x)}+\left(\psi^{\prime \prime}(a)-\eta^{\prime \prime}(a)\right) x \\
& -\left(\frac{\zeta^{\prime \prime}(x) \eta^{\prime}(a) \psi(a)}{\left[\zeta^{\prime}(x)\right]^{2}}-\psi^{\prime}(a)+\eta^{\prime}(a)\right) \frac{d x}{d a} .
\end{aligned}
$$

After inserting $\frac{d x}{d a}$ derived from (2.8), the second order condition $W_{a a}<0$ states

$$
\begin{aligned}
0> & \frac{\eta^{\prime \prime}(a) \psi(a) x+\eta^{\prime}(a) \psi^{\prime}(a) x}{\zeta^{\prime}(x)}+\psi^{\prime \prime}(a) x^{2}-\eta^{\prime \prime}(a) x^{2} \\
& +\left(\psi^{\prime}(a) x-\frac{\eta^{\prime}(a) \psi(a)}{\zeta^{\prime}(x)} \frac{\zeta^{\prime \prime}(x) x}{\zeta^{\prime}(x)}-\eta^{\prime}(a) x\right) \frac{\eta^{\prime}(a)}{\zeta^{\prime}(x)}
\end{aligned}
$$

which is fulfilled if

$$
\left(\psi^{\prime}(a)-\eta^{\prime}(a)\right) x-\frac{\eta^{\prime}(a) \psi(a)}{\zeta^{\prime}(x)} \frac{\zeta^{\prime \prime}(x) x}{\zeta^{\prime}(x)}>0 .
$$

It is seen from $(2.9)$ that the assumption $\frac{\zeta^{\prime \prime}(x) x}{\zeta^{\prime}(x)}>-1$ is sufficient to ensure that this is satisfied for $\left(x_{S}, a_{S}\right)$ which is then the unique solution to the maximization problem. 


\section{A.2. Second Degree Price Discrimination}

Assume that IC and PC are binding. Rewriting these constraints using the specific functional forms yields

$$
\begin{gathered}
x_{1}=\frac{\alpha}{\beta}-\frac{p+\gamma\left(a_{1}-a_{2}\right)}{(1-\lambda) \beta}, \\
x_{2}=\frac{p+\gamma\left(a_{1}-a_{2}\right)}{(1-\lambda) \beta}-\frac{\gamma a_{2}}{\lambda \beta} .
\end{gathered}
$$

Inserting these conditions and the specific functional forms in the profit expression gives

$\pi\left(p, a_{1}, a_{2}\right)=\left(p+\left(a_{1}\right)^{0.5}\right)\left(\frac{\alpha}{\beta}-\frac{p+\gamma\left(a_{1}-a_{2}\right)}{(1-\lambda) \beta}\right)+\left(a_{2}\right)^{0.5}\left(\frac{p+\gamma\left(a_{1}-a_{2}\right)}{(1-\lambda) \beta}-\frac{\gamma a_{2}}{\lambda \beta}\right)$.

The first order conditions with respect to $p, a_{1}$, and $a_{2}$ equal

$$
\begin{gathered}
\left(\frac{\alpha}{\beta}-\frac{p+\gamma\left(a_{1}-a_{2}\right)}{(1-\lambda) \beta}\right)-\frac{p+a_{1}^{0.5}}{(1-\lambda) \beta}+\frac{a_{2}^{0.5}}{(1-\lambda) \beta}=0 \\
\frac{0.5}{a_{1}^{5}}\left(\frac{\alpha}{\beta}-\frac{p+\gamma\left(a_{1}-a_{2}\right)}{(1-\lambda) \beta}\right)-\gamma \frac{\left(p+a_{1}^{0.5}\right)}{(1-\lambda) \beta}+\gamma \frac{a_{2}^{0.5}}{(1-\lambda) \beta}=0 \\
\frac{\gamma\left(p+a_{1}^{0.5}\right)}{(1-\lambda) \beta}+\frac{0.5}{a_{2}^{0.5}}\left(-\gamma \frac{a_{2}}{\lambda \beta}+\frac{p+\gamma\left(a_{1}-a_{2}\right)}{(1-\lambda) \beta}\right)+\left(-\frac{\gamma a_{2}^{0.5}}{\lambda \beta}-\frac{\gamma a_{2}^{0.5}}{(1-\lambda) \beta}\right)=0
\end{gathered}
$$

Dividing (A.4) with (A.3) gives the following solution for the advertising level in the first broadcast,

$$
a_{1}=\frac{0.25}{\gamma^{2}}
$$

which is identical to the advertising level with pay-per-view without price discrimination. Inserting this solution into (A.3) and (A.5) gives

$$
\begin{gathered}
p=\frac{1-\lambda}{2} \alpha-\frac{3}{8 \gamma}+\frac{\gamma}{2} a_{2}+\frac{1}{2} a_{2}^{0.5}, \\
p\left(\gamma a_{2}^{0.5}+\frac{1}{2}\right)+\frac{1}{2} a_{2}^{0.5}+\frac{1}{8 \gamma}-\frac{3}{2} a_{2} \frac{\gamma}{\lambda}=0 .
\end{gathered}
$$

Combining these two equations yields

$$
\begin{aligned}
0= & -8 \lambda\left(\gamma \sqrt{a_{2}}\right)^{3}+12(2-\lambda)\left(\gamma \sqrt{a_{2}}\right)^{2}-(8 \alpha \gamma(1-\lambda)+6) \lambda\left(\gamma \sqrt{a_{2}}\right) \\
& -(4 \alpha \gamma(1-\lambda)-1) \lambda
\end{aligned}
$$


Solutions to $a_{1}, a_{2}, x_{1}, x_{2}$, and $p$ are determined by (A.1), (A.2), (A.6), and (A.7). Afterwards, total surplus under second degree price discrimination may be derived by inserting these solutions into,

$W_{2 .}=\int_{0}^{x_{1}} p(s) d s+\psi\left(a_{1}\right) x_{1}-\eta\left(a_{1}\right) x_{1}+\lambda \int_{x_{1}}^{x_{1}+x_{2}} p(s) d s+\psi\left(a_{2}\right) x_{2}-\eta\left(a_{2}\right) x_{2}$.

After inserting specific functional this equals,

$$
\begin{aligned}
W_{2 .}= & (1-\lambda)\left(\alpha x_{1}-\frac{1}{2} \beta x_{1}^{2}\right)+\left(a_{1}^{0.5}-\gamma a_{1}\right) x_{1} \\
& +\lambda\left(\alpha\left(x_{1}+x_{2}\right)-\frac{\beta}{2}\left(x_{1}+x_{2}\right)^{2}\right)+\left(a_{2}^{0.5}-\gamma a_{2}\right) x_{2} .
\end{aligned}
$$

Finally, relative welfare is derived by,

$$
z(\alpha, \gamma, \lambda)=\frac{W_{2}}{W_{A}}
$$

In order to create Figure 3, we need the following,

Lemma A.1. $z(\alpha, \gamma, \lambda)$ is constant along a hyperbola $\gamma=\rho / \alpha$ for a given value of $\lambda$ where $\rho$ is a positive constant.

Proof. For a given value of $\lambda$, we consider points in the parameter space lying on the hyperbola $\gamma=\rho / \alpha$ where $\rho$ is a constant. It is easy to show from (A.6) and (A.9) that,

$$
a_{1}=\alpha^{2} a_{1}(\rho, \lambda) \quad, \quad a_{2}=\alpha^{2} a_{2}(\rho, \lambda) .
$$

From (A.1), (A.2), (A.7), and the above relationships, we get

$$
\begin{aligned}
p & =\alpha p(\rho, \lambda), \\
x_{1} & =\frac{\alpha}{\beta} x_{1}(\rho, \lambda), \\
x_{2} & =\frac{\alpha}{\beta} x_{2}(\rho, \lambda) .
\end{aligned}
$$

Inserting into (A.10), we get

$$
W_{2 .}=\frac{\alpha^{2}}{\beta} W_{2 .}(\rho, \lambda)
$$


It follows directly from the solution of $W_{A}$ (see Section 4) that

$$
\frac{W_{2}}{W_{A}}=k(\rho, \lambda),
$$

where $k(\rho, \lambda)$ is a constant.

This lemma implies that we only need to compare $z(\alpha, \gamma, \lambda)$ and $f(\alpha, \gamma)$ on the $45^{0}$-line for a given value of $\lambda$. Unfortunately, it is impossible to find closed form solutions to equation (A.9). Therefore, Figure 3 is made by first solving for $a_{2}$ for a given set of parameters using Matematica. Afterwards, the solutions to the other variables are found using (A.1), (A.2), (A.6), and (A.7). Finally, $z(\alpha, \gamma, \lambda)$ is calculated using the above formulas.

To be a solution the following must be satisfied (i) $p \geq 0$ and (ii) $\Pi_{2} \geq \Pi_{P}$. If the first constraint is binding, the solution is identical to the pure advertising solution. This corresponds to the horizontal line at 1 starting the curves in Figure 3. If the second condition is binding, the solution is identical to the pay-per-view solution without price discrimination. This occurs to right of the intersections between the dashed curves and the solid curve. 
Figure la

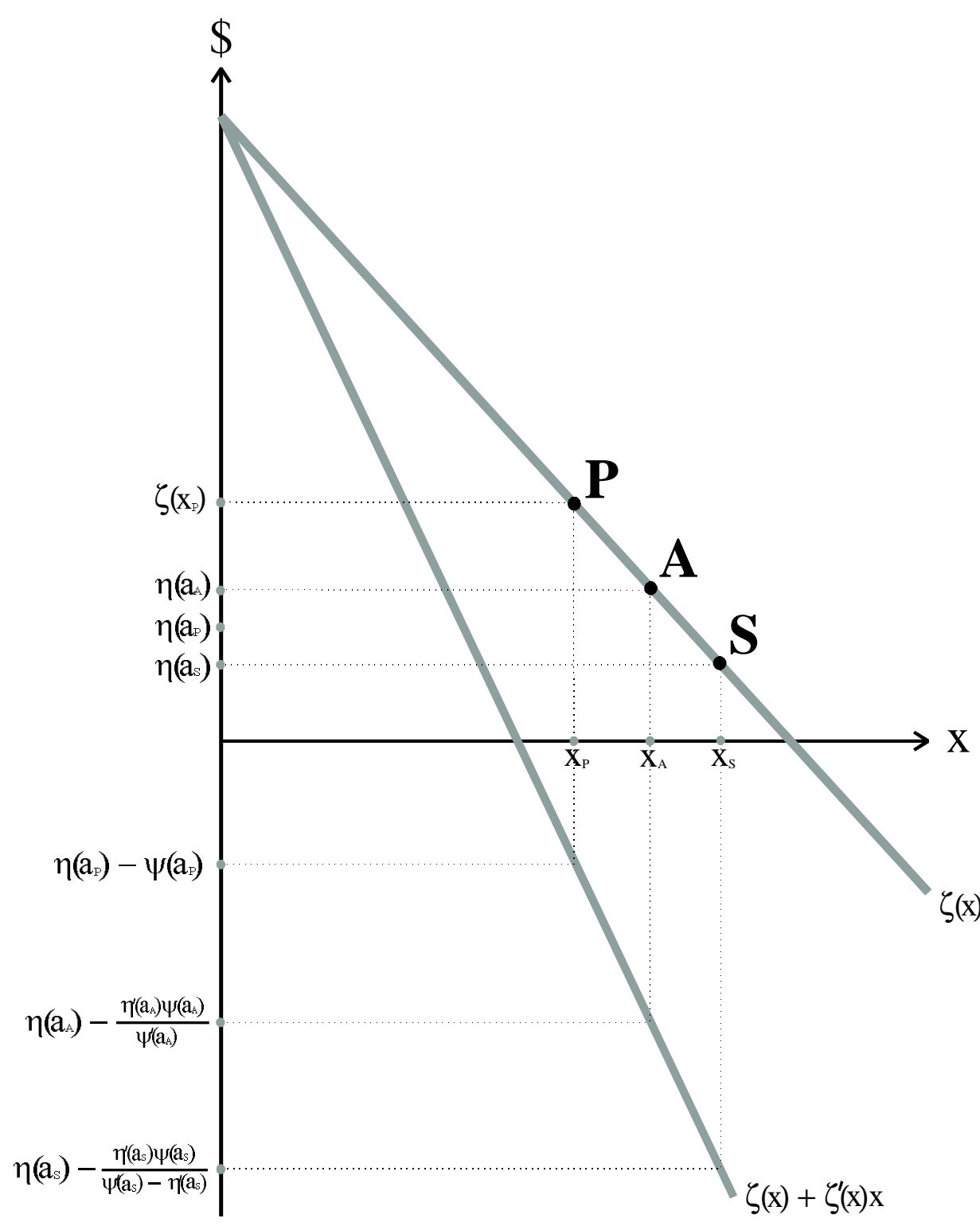

Figure $1 b$

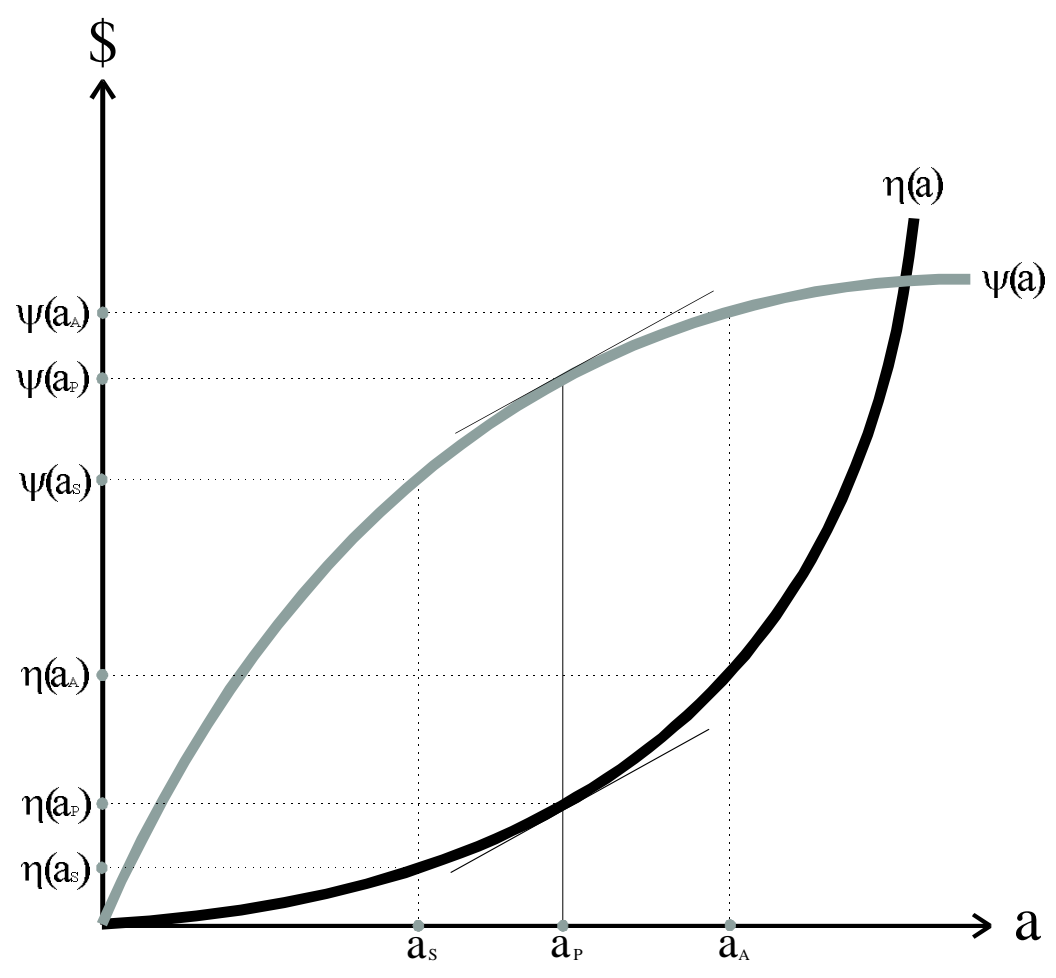


Figure 2

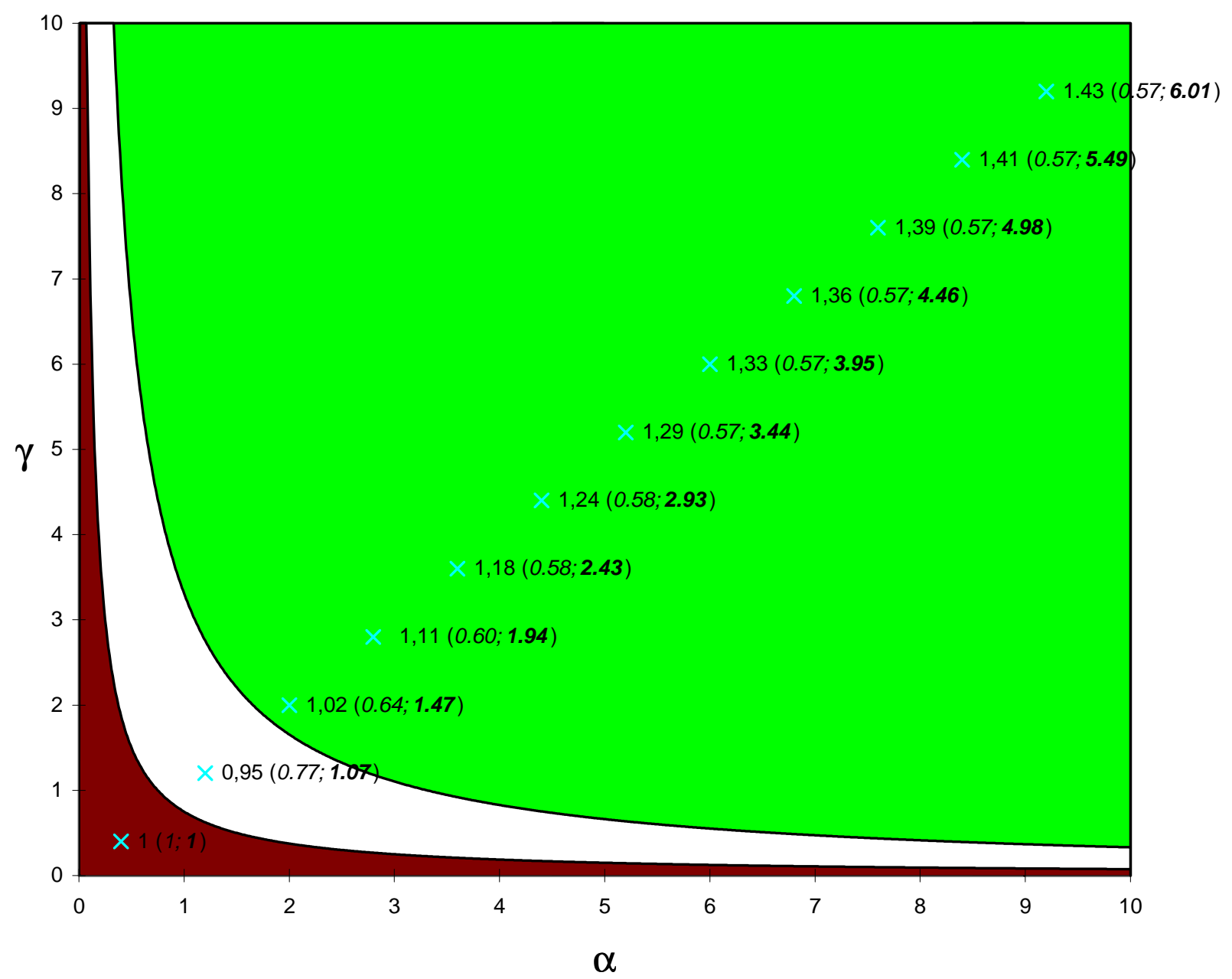

$\square$ Identical Welfare $\square$ Advertising $\square$ Pay-per-view 
Figure 3

Pay-per-view Vs. Advertising with Standard Price

Setting and Second Degree Price Discrimination

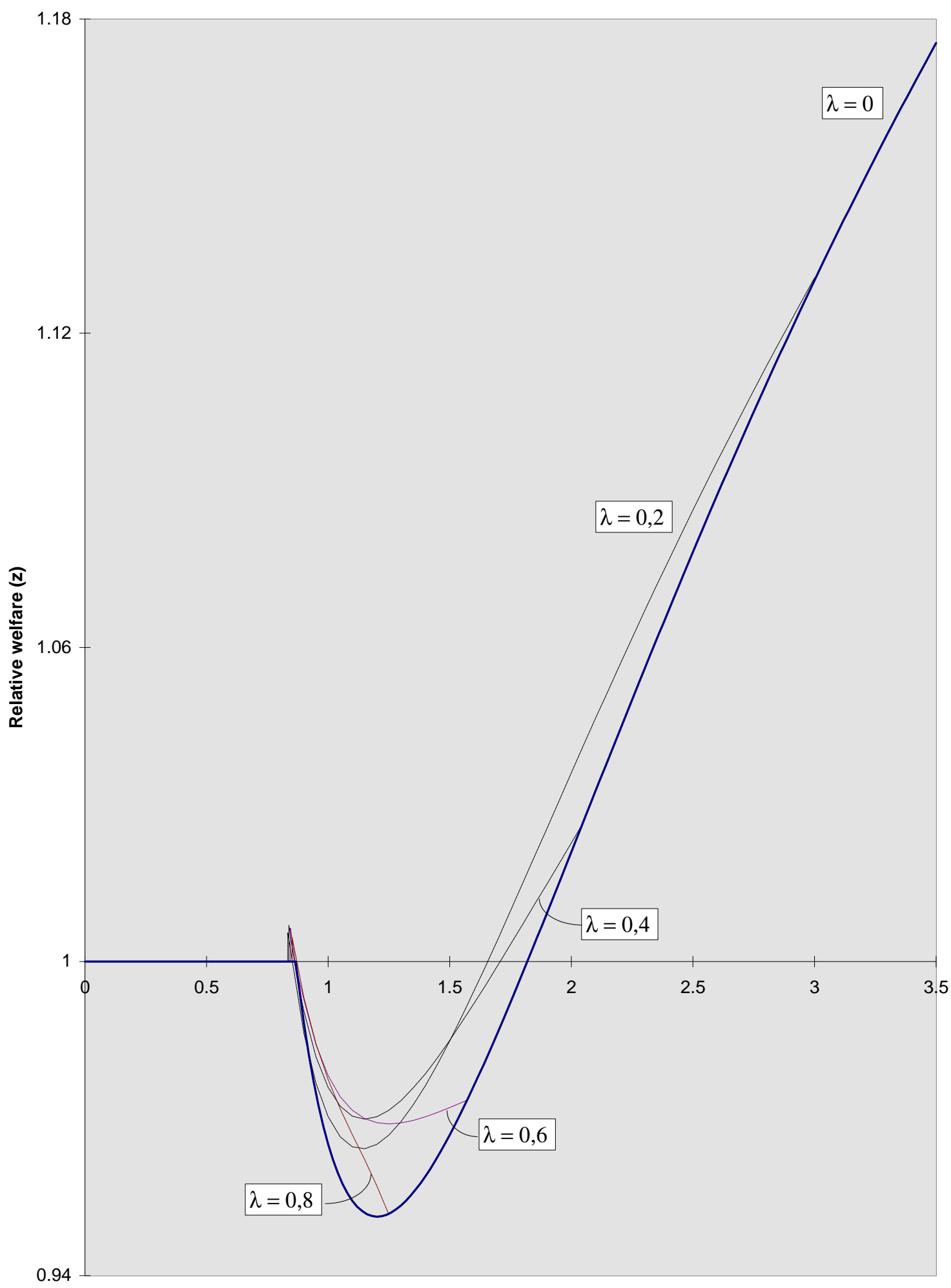

$$
\alpha=\gamma
$$

